\title{
THE ORDER OF THE AUTOMORPHISM GROUP OF A CENTRAL PRODUCT
}

\author{
KENNETH G. HUMMEL 1
}

ABSTRACT. If a $p$-group $G$ is the central product of nontrivial subgroups $H$ and $A$, where $A$ is abelian and $o(H) \mid o($ Aut $H)$, then $o(G) \mid o($ Aut $G)$.

1. Introduction. There has been interest in recent years in the conjecture that if $G$ is a noncyclic $p$-group of order $o(G)=p^{n}$, with $n \geq 3$, then $o(G) \mid o$ (Aut $G$ ). The conjecture has been established for $p$-groups of class 2 [2] and for $p$-abelian $p$-groups [1]. Otto [3] has shown that if $G=A \times B, A$ is abelian and $o(B) \mid o($ Aut $B$ ), then $o(G) \mid o$ (Aut $G$ ). A result of this type not only extends the number of groups for which the conjecture is known to be true, but also, and perhaps more importantly, shows that the truth of the overall conjecture depends only on being able to prove it for a smaller class of groups. Otto's result shows that it is sufficient to consider $p$-groups with no nontrivial abelian direct factors.

It is the purpose of this paper it prove that if a $p$-group $G$ is the central product of subgroups $H$ and $A$, where $A$ is abelian and $o(H) \mid o$ (Aut $H$ ), then $o(G) \mid o$ (Aut $G$ ). It might be noted here that this result serves the same two purposes as Otto's result, and further that Otto's result is a special case of this theorem.

Throughout this paper the following notations are used. $o(G)$ is the order of $G$; Aut $G$ is the automorphism group of $G$; for $a \in G,\langle a\rangle$ denotes the subgroup generated by $a ; Z(G)$ is the center of $G$; $A{ }^{A}{ }_{H} G$ is the group of automorphisms of $G$ leaving $H$ invariant; $\Omega_{1}(G)=\langle\{x \in G: o(x) \mid p\}\rangle ; \mathcal{O}_{1}(G)=$ $\left\langle\left\{x^{p}: x \in G\right\}\right\rangle ; \operatorname{St}(a)$ is the stabilizer of $a ;|G: H|$ is the index of $H$ in $G$; and $|G|_{p}=p^{n}$, where $p^{n} \mid \rho(G)$, but $p^{n+1} \dagger o(G)$.

2. Extendable automorphisms. We begin by proving the following lemma, which is of some interest in its own right.

Presented to the Society, January 18, 1974 under the title On the order of the automorphism group of the central product; received by the editors January 14, 1974. AMS (MOS) subject classifications (1970). Primary 20D15, $20 \mathrm{D} 45$.

Key words and phrases. Central product, automorphism group, p-group.

1 The author gratefully acknowledges the assistance of Dr. Joseph Buckley in the development of this paper. 
Lemma 1. If $G=\langle a\rangle H, a \in Z(G)$ and $H$ is a nontrivial maximal subgroup of the p-group $G$, then $a \in$ Aut $H$ extends to $\bar{\alpha} \in$ Aut $G$ if and only if $\alpha\left(a^{p}\right)=a^{s p} h^{p}$, where $0<s<p$ and $h \in Z(H)$.

Proof. Let $a \in$ Aut $H$ be extendable to $\bar{\alpha} \in$ Aut $G$. Noting each element $g \in G$ can be expressed uniquely in the form $g=a^{k} h, 0 \leq k<p, b \in H$, we see $\bar{\alpha}(a)=a^{s} h, 0 \leq s<p, h \in H$. But $s \neq 0$, for otherwise $\bar{\alpha}$ would map $G$ into $H$ and not be onto. Also, $a \in Z(G)$ implies $\bar{\alpha}(a)=a^{s} h \in Z(G)$, and so $h \in H \cap Z(G)=Z(H)$. Hence $a\left(a^{p}\right)=[\bar{a}(a)]^{p}=\left(a^{s} h\right)^{p}=a^{s p} h^{p}, 0<s<p$, $h \in Z(H)$. Conversely, let $a \in$ Aut $H$ and $a\left(a^{p}\right)=a^{s p} b^{p}, 0<s<p, h \in Z(H)$, and define $\bar{\alpha}(g)=\bar{\alpha}\left(a^{k} h^{\prime}\right)=a^{s k} b^{k} \alpha\left(b^{\prime}\right)$. To see that $\bar{\alpha}$ is a homomorphism, let $g_{1}, g_{2} \in G$. Then $g_{1}=a^{k_{1}} h_{1}, g_{2}=a^{k_{2}} b_{2}$, where $0 \leq k_{1}, k_{2}<p$ and $h_{1}$, $h_{2} \in H$. Let $k_{1}+k_{2}=k_{3}+r p$, where $0 \leq k_{3}<p$ and $r \geq 0$. Then

$$
\begin{aligned}
\bar{\alpha}\left(g_{1} g_{2}\right) & =\bar{\alpha}\left(a^{k} a^{r p} h_{1} h_{2}\right)=a^{s k_{3}} h^{k_{3}} \alpha\left(a^{r p}\right) \alpha\left(h_{1}\right) \alpha\left(h_{2}\right) \\
& =a^{s k} h^{k} a^{s r p} h^{r p} \alpha\left(h_{1}\right) \alpha\left(h_{2}\right)=a^{s\left(k_{1}+k_{2}\right)} h^{k_{1}+k_{2}} \alpha\left(h_{1}\right) \alpha\left(h_{2}\right) \\
& =a^{s k} h^{k}{ }^{k} \alpha\left(h_{1}\right) a^{s k_{2}} h^{k_{2}} \alpha\left(h_{2}\right)=\bar{\alpha}\left(g_{1}\right) \bar{\alpha}\left(g_{2}\right) .
\end{aligned}
$$

Clearly $\bar{\alpha}$ extends $\alpha$; further $\bar{\alpha}\left(a^{k}{ }^{1} h_{1}\right)=\bar{\alpha}\left(a^{k} h_{2}\right)$ implies $a^{s k}{ }^{k} b^{k}{ }^{1} \alpha\left(h_{1}\right)=$ $a^{s k_{2}} b^{k_{2}} a\left(b_{2}\right)$, and hence $a^{s\left(k_{1}-k_{2}\right)} \in H$, so $k_{1}=k_{2}$. It follows that $a\left(h_{1}\right)$ $=a\left(h_{2}\right)$ and hence $h_{1}=h_{2}$. Hence $\bar{\alpha}$ is $1-1$ and $\bar{\alpha} \in$ Aut $G$.

3. The order of Aut $G$. We first establish a special case of the main theorem, from which the general result follows quite easily.

Lemma 2. If $G=\langle a\rangle H, a \in Z(G), H$ is a nontrivial maximal subgroup of the p-group $G$, and $o(H) \mid o($ Aut $H)$, then $o(G) \mid o\left(\right.$ Aut $\left._{H} G\right) \mid o($ Aut $G)$.

Proof. Case 1. $o(a)=p$. This is actually a special case of Otto's result. However we give an independent proof so that Otto's result might be seen to be a corollary of theTheorem. We first note that every automorphism of $H$ extends to an automorphism of $G$. Hence the restriction map $\theta:$ Aut $_{H} G$ $\rightarrow$ Aut $H$ is onto.

Let $a \in \operatorname{ker} \theta$. We must have $\alpha(a)=a^{s} h$, where $0<s<p$ and $h \epsilon$ $\Omega_{1}(Z(H))$. Conversely, for each $s, 0<s<p$, and each $h \in \Omega_{1}(Z(H))$, define $\beta: G \rightarrow G$ (dependent on the choices of $s, h)$ by $\beta\left(a^{k} h_{1}\right)=a^{s k} b^{k} h_{1}$. Then $\beta \in \operatorname{ker} \theta$. Hence, $o(\operatorname{ker} \theta)=(p-1) o\left(\Omega_{1}(Z(H))\right)$. We conclude that

$$
o\left(\text { Aut }_{H} G\right)=d(\operatorname{ker} \theta) o(\text { Aut } H)=(p-1) o\left(\Omega_{1}(Z(H))\right)_{o}(\text { Aut } H) \text {, }
$$


and since $o(H) \mid \alpha\left(\right.$ Aut $H$ ), we see $o(G) \mid \propto\left(\right.$ Aut $\left._{H} G\right) \mid \alpha($ Aut $G$ ).

Case 2. $\alpha(a)>$ p. A restatement of Lemma 1 shows that $\alpha \in$ Aut $H$ extends to $\bar{\alpha} \in$ Aut $G$ if and only if the induced automorphism $\alpha^{*} \epsilon$ Aut $\left[Z(H) / \mathcal{O}_{1}(Z(H))\right]$ leaves $\left\langle\bar{a}^{p}\right\rangle \leq Z(H) / \Theta_{1}(Z(H))$ invariant. Consider the permutation representation (Aut $H, X)$, where $X=$ \{yclic subgroups of $\left.Z(H) / \Theta_{1}(Z(H))\right\}$. Then $E=\{\alpha \in$ Aut $H: \alpha$ is extendable to $\bar{\alpha} \in$ Aut $G\}=$ St $\left(\left\langle\bar{a}^{p}\right\rangle\right)$. Now $\mid$ Aut $H: E \mid$ is the order of one orbit, and hence

$$
\mid \text { Aut } H: E \mid \leq d(X)=\left(p^{r}-1\right) /(p-1)<p^{r},
$$

where $Z(H) / \bigcirc_{1}(Z(H))$ is elementary abelian of rank $r$. Hence $p^{r}>$ $\mid$ Aut $\left.H\right|_{p} /|E|_{p}$ and $\mid$ Aut $\left.\left.H\right|_{p}\left|p^{r-1}\right| E\right|_{p^{*}}$ Using the hypothesis that $\alpha(H) \mid \propto($ Aut $H)$, we see $\left.\alpha(G)\left|p^{r}\right| E\right|_{p}$, and we are finished if $p^{r}|E|_{p} \mid \propto\left(\right.$ Aut $\left._{H} G\right)$. Consider the restriction map $\theta:$ Aut $_{H} G \rightarrow$ Aut $H$, and note that $\operatorname{Im} \theta=E$. Let $a \in \operatorname{ker} \theta$. Suppose $a(a)=a^{s} h$, where $0<s<p$ and $h \in Z(H)$. Then $\alpha\left(a^{p}\right)=[a(a)]^{p}=a^{p}$ implies $a^{s} h \equiv a \bmod \Omega_{1}(Z(G))$. Clearly, $\Omega_{1}(Z(H)) \leq$ $\Omega_{1}(Z(G))$. If this inclusion is proper, there exists an element $a^{\prime} \in Z(G)$ with $a^{\prime} \notin H$ and $o\left(a^{\prime}\right)=p$. Hence Case 1 applies. Hence without loss of generality, $\Omega_{1}(Z(H))=\Omega_{1}(Z(G))$, and con sequently $a^{s} h=a h^{\prime}$ for some $h^{\prime} \epsilon$ $\Omega_{1}(Z(H))$. Conversely for each $h^{\prime} \in \Omega_{1}(Z(H))$, the map $\bar{\alpha}: G \rightarrow G$ given by $\bar{a}\left(a^{k} h\right)=\left(a h^{\prime}\right)^{k} b$ is in $\operatorname{ker} \theta$. Hence

$$
o(\operatorname{ker} \theta)=o\left(\Omega_{1}(Z(H))\right)=d Z(H) / \mathcal{O}_{1}(Z(H))=p^{r} .
$$

But Aut ${ }_{H} G /$ ker $\theta \simeq E$, and hence $p^{r} o(E)=o\left(\right.$ Aut $\left._{H} G\right)$, which implies that $p^{r}|E|_{p} \mid \partial\left(\right.$ Aut $\left._{H} G\right)$ completing the proof.

We are now able to prove the

Theorem. If a p-group $G$ is the central product of nontrivial subgroups $H$ and $A$, where $A$ is abelian and $o(H) \mid o($ Aut $H)$, then $o(G) \mid o$ (Aut $G$ ).

Proof. Note $A \leq Z(G)$ and let $|A: H \cap A|=p^{n}$, with $n \geq 1$. The proof is by induction on $n$. If $n=1$, then Lemma 2 applies, for without loss of generality $A=\langle a\rangle$. For $n\rangle 1$, select $A_{1} \leq A$ such that $H \cap A<A_{1}<A$ and $\left|A_{1}: H \cap A\right|=p$. Let $H_{1}=H A_{1}$. Then $H \cap A_{1}=H \cap A$ and $\left|A_{1}: H \cap A_{1}\right|=p$. Hence by Lemma 2, o $\left(H_{1}\right) \mid \propto\left(\right.$ Aut $\left.H_{1}\right)$. But $G=H_{1} A$ and $H_{1} \cap A=A_{1}$, hence $\left|A: H_{1} \cap A\right|=p^{n-1}$, and the induction hypothesis yields $o(G) \mid o$ (Aut $G$ ).

It might be noted here that for a $p$-group $G$, the following are equivalent: (1) $G$ is the central product of proper subgroups $A$ and $H$ where $A$ is is abelian; (2) there exists a maximal subgroup $K$ of $G$ such that $Z(K)<$ $Z(G)$; (3) $Z(G) \unrhd \Phi(G)$. Hence in trying to establish the conjecture, one can restrict attention to $p$-groups $G$ for which $Z(G) \leq \Phi(G)$. 


\section{REFERENCES}

1. R. Davitt, The automorphism group of finite $p$-Abelian p-groups, Illinois J. Math. 16 ( 1972), 76-85. MR 46 \#7378.

2. R. Faudree, $A$ note on the automorphism group of a p-group, Proc. Amer. Math. Soc. 19 (1968), 1379-1382. MR 40 \#1476.

3. A. D. Otto, Central automorphisms of a finite p-group, Trans. Amer. Math. Soc. 125 (1966), 280-287. MR 34 \#4362.

DEPARTMENT OF MATHEMATICS, WESTERN MICHIGAN UNIVERSITY, KALAMAZOO, MICHIGAN 49001

Current address: Department of Mathematics, Illinois State University, Normal, Illinois 61671 